\title{
Epidemiological Characteristics of 417 patients infected with COVID-19 and 368 discharged cases among them in Shenzhen City, China
}

Bo Yuan

Science and education department, Shenzhen Samii Medical Center

\section{Ya-Wen An}

Science and education department, Shenzhen Samii Medical Center

\section{Yong-Xin Chen}

Science and education department, Shenzhen Samii Medical Center

Jing Yang

Quality control department, the Third People's Hospital of Shenzhen

Jian-Chun Wang

Science and education department, Shenzhen Samii Medical Center

Wei-Xin Li

Science and education department, Shenzhen Samii Medical Center

Cheng Wang

Science and education department, Shenzhen Samii Medical Center

Shuo Song ( $\nabla$ songshuo@ssmc-sz.com )

Science and education department, Shenzhen Samii Medical Center

Han-Qing Liu ( $D$ liuhanqing@ssmc-sz.com)

Science and education department, Shenzhen Samii Medical Center

\section{Research Article}

Keywords: COVID-19, Shenzhen city, clinical characteristics, outcomes

Posted Date: March 26th, 2020

DOl: https://doi.org/10.21203/rs.3.rs-19554/v1

License: (c) (i) This work is licensed under a Creative Commons Attribution 4.0 International License.

Read Full License 


\section{Abstract}

The outbreak of Coronavirus Disease 2019 (COVID-19) is getting worse every day all over the world. The present study aimed to review the epidemiological characteristics of patients infected with COVID-19 in Shenzhen city, a super megacity of China, to provide some references for fighting to the coronavirus. We collected data of 417 patients with laboratory-confirmed COVID-19 of Shenzhen through March $7^{\text {th }}, 2020$. The epidemiological characteristics of the patients were analyzed. Besides, we collected the governmental measures of Shenzhen city, and the the dynamic changes of the epidemic outbreak. Governmental strategies such as early detection, early hospitalization and popular science etc. are effective for the prevention and control of the epidemic. Nearly $80 \%$ confirmed patients with COVID-19 in Shenzhen were in normal or mild conditions, and the mortality was less than $1 \%$. Age, gender, exposure to source of transmission within 14 days and basic diseases are major risk factors for severe patients. Patients in characters of elder, male, with exposure of Wuhan and carried basic diseases had higher risk to be in severe condition ( $P<0.01 ; P<0.001)$. There were 368 patients discharged from hospital by the end

of March $7^{\text {th }}, 2020$. Patients in severe conditions took more time from onset to discharge $(P<0.001)$, so as those elder one $(P<0.001)$ or who carried basic diseases $(P<0.01)$. The differences of epidemiological characteristics between children and the elderly are still not clear. The nucleic acid test of some discharged patients returned to positive again, and the potential mechanisms need to be further explored. In conclusion, timely administrative intervention is necessary for prevention and control of the COVID-19 outbreak. Patients in characters of elder, male and carried basic diseases worthy more attention. The management of discharged patients, especially the investigation of the recurrence of positive SARS-CoV2 RNA will be the focus on for the next step.

Authors Bo Yuan and Ya-Wen An contributed equally to this work.

\section{Introduction}

In early December 2019, a novel coronavirus named SARS-COV-2 started spreading in Wuhan, China. At present, the Coronavirus Disease 2019 (COVID-19) induced by SARS-COV-2 has been basically controlled in China ${ }^{1}$. Daily new confirmed cases of COVID-19 nationwide except Hubei province dropped to zero on March $8^{\text {th }}$. Unfortunately, we noticed that worldwide outbreak of COVID-19 started, and more than 200,000 infected persons were confirmed involving nearly every countries and regions all over the world. People of the world need more guidance to fight to the coronavirus ${ }^{2}$. Outbreak of COVID-19 in Wuhan got a lot of attention at past ${ }^{3,4}$, but there are still lack of researches about that in other cities of China.

Shenzhen is one of the economic centers of China. The managed population (permanent plus mobile population) of Shenzhen in the end of 2018 was more than 25 million. Hubei province is one of the main sources of Shenzhen's population ${ }^{5}$, during January $15^{\text {th }}$ to January $23^{\text {rd }}$ (before Wuhan closed), Shenzhen became the No.1 destination city outside Hubei for people left Wuhan. Therefore, the outbreak of COVID-19 in Shenzhen is very typical and significative for other big cities of the world to prevent the 
epidemic. The present study aimed to review the outbreak of COVID-19 in Shenzhen. Total of 417 local laboratory-confirmed COVID-19 patients and 368 discharged cases among them were analyzed.

\section{Methods}

\section{Data sources}

All the data was obtained from public disclosure of Shenzhen Municipal Health Commission through March $7^{\text {th }}$. This study was approved by the Ethics Committee of Shenzhen Samii Medical Center and written informed consent was waived in light of the urgent need to collect clinical data. We declare that these data do not involve any private information of patients. It is worth pointing out that imported COVID-19 cases from overseas were not included in the scope of this analysis.

\section{Statistical analysis}

Patients infected with COVID-19 were divided into severe and non-severe groups according to the Guidelines for diagnosis and treatment of National Health Commission of the People's Republic of China. Continuous variables were expressed as the means and standard deviations and one-way ANOVA test were used for inter-group compare. Categorical variables were summarized as the counts and percentages in each category and compared using Chi-square test. Pearson test was used for correlation analysis. The candidate risk factors included age, gender, exposure history and basic diseases were included into the final models. All analyses were conducted with SPSS software version 19.0.

\section{Results}

Since the first patients infected with COVID-19 of Shenzhen was confirmed, the government immediately took a series of measures such as hospital confinement, traffic screening, extending vacations, postponing the reopening time of schools, offering free nucleic acid testing, $100 \%$ closed community management, etc.,, Figure-1A. On February $21^{\text {st }}$, the total number of discharged patients exceeded that of hospitalized patients for the first time, Figure-1B. The daily updated number of close contacts and hospitalized patients in critical and severe conditions continued to decline, Figure $1 C$ and $D$.

All confirmed patients were treated at the Third People's Hospital of Shenzhen. Among the 417 local confirmed cases, there were $198(47.5 \%)$ male and $219(52.5 \%)$ female. The ages were ranged $1-86$ years old, averaged at $45.4 \pm 17.7$ years old, the median age and interquartile range was $47(34-60)$ years old, Figure $2 A$. All the numbers were significantly less than previous reports 5,6 . The number of patients has mild, normal, severe and critical symptoms was 16(3.8\%), 309(74.1\%), $73(17.5 \%)$ and $19(4.6 \%)$, respectively. All of the three fatalities were males over 60 years old, and the case fatality ratio $(0.72 \%$, $3 / 417$ ) was equal to the national average ${ }^{6}$. To facilitate statistics, we combined mild and normal patients into non-severe group, and severe and critical patients into severe group. 
Age, gender, exposure to source of transmission within 14 days and basic diseases are major risk factors for severe patients, Table 1. The average ages of severe patients were over 16 years elder than non-severe patients $(P<0.001)$. The ratio of patients under 18 years old in Shenzhen reached $7.2 \%$, which was significantly higher than the national level, fortunately, there were no severe patients in children, the proportion of severe patients rapidly increased with age, nearly half (48.9\%) of elders over or equal to 66 years old were in severe conditions.

The proportion of severe patients in male were almost twice than in female $(64.1 \%$ vs $35.9, \mathrm{P}<0.001)$. Over half (53.2\%) of the 417 patients has Wuhan exposure history in the last 14 days before their selfnarrative onset day. The median onset date for patients with Wuhan exposure was 5 days' earlier than those with no Wuhan exposure (January $24^{\text {th }} v s$ January $29^{\text {th }}$ ), Figure $2 B$. Twenty-two out of the 417 cases have no clear contact history (never left Shenzhen, or contact any infected patient), it suggests that there's risk of active vital transmission in Shenzhen. Patients who exposed to Wuhan before onset within 14 days has higher severe proportion than those with no Wuhan exposure $(27.5 \%$ vs $15.9 \%, \mathrm{P}<0.01)$, Table 1.

Patients carried at least on basic diseases were under higher risks too ( $36.4 \%$ vs $16 \%, \mathrm{P}<0.001)$. Metabolic diseases were the most common basic diseases carried by the confirmed cases. Patients carried with hypertension, diabetes, hyperlipidemia, cardiovascular and cerebrovascular diseases and malignant tumors have higher risks of developing severe condition. Among that, the ratio of severe was highest (60.7\%) in patients with cardiovascular and cerebrovascular diseases, Table 1.

Obvious family clusters were observed in the 417 confirmed cases of Shenzhen involving 84 clusters totally $229(54.9 \%)$ infected patients, it is much higher than in previous studies ${ }^{7,8}$. The first familial cluster was shown in Figure 2C, case 5 was the first medical patients in Guangdong province and become the key person to find out the first familial cluster; case 1 was the first confirmed patients in Guangdong, and died on February 16. In addition to case 5 , the other four have a history of exposure to Wuhan. The largest local community cluster of cases was shown in Figure 2D, all the 9 cases have no Wuhan exposure history.

Totally of 368 patients have been discharged from the hospital by the end the March $7^{\text {th }}$, the basic information was shown in Table 1. The proportion of age, gender, exposure history and basic diseases were similar to the total 417 confirmed patients.

As shown in Table 2, the days of hospitalization showed no significant difference between men and women. Patients with Wuhan exposure history seemed to need more time of hospitalization, but there was no significant difference compared to those with no exposure. Age was a main influence factor for discharge. Patients $\leq 45$ years old takes less time $(P<0.001)$ from onset to discharge than elder ones ( $\geq 46$ years old). Pearson test showed that ages of patients was positively correlated with the time from onset to admission $(0.125, P=0.017)$, admission to discharge $(0.273, P=0.000)$ and onset to discharge $(0.301, P=0.000)$. The patients carried at least one basic disease took longer time of hospitalization than 
those with no basic diseases $(\mathrm{P}<0.05)$. And the patients in severe conditions spend more time in hospital than those in non-severe conditions $(P<0.001)$. Besides, we noticed that patients in severe conditions went to hospital later than those in non-severe conditions $(P<0.05)$, and that might be partly influenced the progression of disease.

\section{Discussion}

Intensive measures for epidemic prevention and control were signed by the government of Shenzhen city such as hospital confinement, traffic screening, extending vacations, postponing the reopening time of schools, offering free nucleic acid testing, $100 \%$ closed community management, etc. since January $19^{\text {th }}$, when the first patients infected with COVID-19 of Guangdong province were laboratory-confirmed. The governmental actions were helpful for controlling the COVID-19 outbreak in China, this is in line with a series of recently reported studies ${ }^{9,10}$. However, some studies have proposed the opposite view ${ }^{11}$, larger sample studies are needed in future. In addition, it remains to be observed whether the Chinese strategies work elsewhere ${ }^{12}$.

For now, adolescents seemed less susceptible to SARS-COV-2 and there was no severe case under 18 years old in Shenzhen. However, since most cases in children were detected by tracing close contacts of families with adult cases, the susceptibility and infectivity are still unclear. Home confinement of children is helpful for preventing outbreak of COVID-19, however, it might affect the their psychology and physiology ${ }^{13}$, the situation might worsen accompanied by their parents return to work. How to mitigate the effects need to pay more attention. Besides, we noticed a very strange phenomenon, the number of girls under 18 years old was almost twice of boys (19/11), however, the ratio of women to men has reversed (16/29) for elderly patients over 65 years old, which has never been reported before.

Persons in characters of male, elder than 65 years old and carried basic diseases should pay more attention to protect their selves since the higher chance to be in severe conditions once infected with COVID-19, especially for those who carried metabolic diseases and cardiovascular diseases. The longer time took from onset to discharged indicated the managements of patients in severe conditions is still facing challenges. Besides, we noticed that, 12 out of 417 (2.9\%) confirmed cases with history of liver diseases, among them, there were 3 patients with fatty liver and 9 patients carried hepatitis $B$ virus. Although the proportion in severe conditions and discharged time for patients with liver diseases were similar to the whole sample, the liver injuries attributed to direct virus attack to liver and drug-induced liver injuries and inflammatory responses should be noticed in the managements. Zhang et al ${ }^{14}$ found that $14-53 \%$ of COVID-19 patients have elevated levels of alanine aminotransferase and aspartic aminotransferase during disease progression.

For China, the management of discharged patients might be strengthened in future. The discharge standard in China for now is: (1) body temperature return to normal level for more than 3 days after clinical rehabilitation, (2) the clinical symptoms disappear and the imaging performance is significantly improved, and (3) twice negative of nucleic acid detection interval of 24 hours. However, recently, nucleic 
acid detections of many discharged patients were positive again and the reasons are still not clear. Of particular note is that, Shenzhen government request the discharged patients undergoing another 14days' confinement and medical observations in an assigned place since February $22^{\text {nd }}$. So far, the nucleic acid detections of throat swab or anal swab for more than $10 \%$ of discharged patients in Shenzhen have recovered positive. We will work with the Shenzhen Center for Disease Control and Prevention to make further observation on these patients and find out the potential mechanisms. Once the patients were detected positive of throat or anal swab, they will be immediately sent back to the Third People's hospital of Shenzhen for medical observation until they meet discharge criteria again. Despite existing evidence that these patients are not infectious as there is no detectable live virus in their body. The discharge management mode in Shenzhen is worth to be popularized to prevent potential risks.

\section{Declarations}

\section{Acknowledgements}

This study was financially supported by grants JCYJ20180302153611416 from the Science and Technology Planning Project of Shenzhen.

\section{Author Contributions}

B. Y. and Y. W. A. was responsible for data analysis and chart making. Y. X. C., J.Y W. X. L., and C. W. participated in data collection. J. C. W. contributed to the production of Figure 1. S. S. and H. Q. L. drafted and edited the manuscript. All authors reviewed the manuscript and approved the submission.

\section{Availability of data and materials}

The datasets used and/or analyzed during the current study are available from the corresponding author on reasonable request.

\section{Competing interest}

The authors declare that they have no competing interest.

\section{Tables}

Table 1. Epidemiological Characteristics of 417 patients with COVID-19 and 368 discharged one among them in Shenzhen 


\begin{tabular}{|c|c|c|c|c|}
\hline \multirow[t]{2}{*}{ Epidemiological Characteristics } & \multicolumn{3}{|c|}{ All confirmed patients } & \multirow{2}{*}{$\begin{array}{l}\text { Discharged } \\
\text { patients }\end{array}$} \\
\hline & $\begin{array}{c}\text { Total } \\
(\mathrm{n}=417)\end{array}$ & $\begin{array}{c}\text { Non-Severe } \\
(n=325)\end{array}$ & $\begin{array}{l}\text { Severe } \\
(n=92)\end{array}$ & \\
\hline Age, Average \pm SD -yrs & $45.4 \pm 17.7$ & $41.8 \pm 17.4$ & $58.1 \pm 11.7^{* * *}$ & $44.5 \pm 17.6$ \\
\hline \multicolumn{5}{|l|}{ Age groups-No., \% } \\
\hline $1-17$ yrs & $30 / 417(7.2)$ & $30 / 30(100)$ & $0 / 92(0)$ & $27 / 368(7.3)$ \\
\hline $18-45$ yrs & $165 / 417(39.6) \quad 1$ & $152 / 165(92.1)$ & $13 / 165(7.9)$ & 153/368(41.6) \\
\hline $46-65$ yrs & $177 / 417(42.4) \quad 1$ & $120 / 177(67.8)$ & $57 / 177(32.2)$ & $152 / 368(41.3)$ \\
\hline$\geq 66$ yrs & $45 / 417(10.8)$ & $23 / 45(51.1)$ & $22 / 45(48.9)$ & $36 / 368(9.8)$ \\
\hline \multicolumn{5}{|l|}{ Gender groups-No., \% } \\
\hline Female & $219 / 417(52.5) \quad 1$ & $186 / 219(57.2)$ & $\begin{array}{l}\text { 33/92(35.9) } \\
\# \# \#\end{array}$ & 194/368(52.7) \\
\hline Male & $198 / 417(47.5) \quad 1$ & $139 / 325(42.8)$ & $59 / 92(64.1)$ & $174 / 368(47.3)$ \\
\hline \multicolumn{5}{|l|}{$\begin{array}{l}\text { Exposure to source within } 14 \\
\text { days- N., \% }\end{array}$} \\
\hline Wuhan exposure & $222 / 417(53.2) 1$ & $161 / 222(72.5)$ & 61/222(27.5) & 196/368(53.3) \\
\hline No Wuhan exposure & $195 / 417(46.8) 1$ & 164/195(84.1) & 31/195(15.9) & $172 / 368(46.7)$ \\
\hline Local residents of Wuhan & $107 / 417(25.7) \quad 7$ & $71 / 325(21.8)$ & 36/92(39.1) & $96 / 368(26.1)$ \\
\hline Recently been to Wuhan & $100 / 417(24.0)$ & $80 / 325(24.6)$ & $20 / 92(21.7)$ & $89 / 368(24.2)$ \\
\hline $\begin{array}{r}\text { Contacted with people from } \\
\text { Wuhan }\end{array}$ & $15 / 417(3.6)$ & 10/325(3.1) & $5 / 92(5.4)$ & 11/368(3.0) \\
\hline Confirmed cases & 108/417(25.9) & 93/325(28.4) & 15/92(16.3) & $91 / 368(24.7)$ \\
\hline Others & $87 / 417(20.9)$ & 71/325 (21.7) & 16/92(17.4) & $81 / 368(22.0)$ \\
\hline \multicolumn{5}{|l|}{ Basic diseases- N., \% } \\
\hline Carried at least one basic diseases & $129 / 417(30.9)$ & $82 / 129(63.6)$ & $\begin{array}{c}47 / 129(36.4) \\
\$ \$ \$\end{array}$ & $108 / 368(29.3)$ \\
\hline No basic diseases & $288 / 417(69.1)$ & ) $242 / 288(84.0)$ & $46 / 288(16.0)$ & $260 / 368(70.6)$ \\
\hline Hypertension & $63 / 417(15.1)$ & $37 / 63(58.7)$ & $26 / 63(41.3)$ & $53 / 368(14.4)$ \\
\hline Diabetes & $32 / 417(7.7)$ & $16 / 32(50.0)$ & $16 / 32(50.0)$ & 25/368(6.8) \\
\hline Hyperlipidemia & $6 / 417(1.4)$ & $2 / 6(33.3)$ & $4 / 6(66.7)$ & $5 / 368(1.4)$ \\
\hline $\begin{array}{r}\text { Cardiovascular and cerebrovascular } \\
\text { diseases }\end{array}$ & $28 / 417(6.7)$ & $11 / 28(39.3)$ & $17 / 28(60.7)$ & $23 / 368(6.3)$ \\
\hline Malignant tumors & $6 / 417(1.4)$ & $4 / 6(66.7)$ & $2 / 6(33.3)$ & 6/368(1.6) \\
\hline Chronic liver disease & $12 / 417(2.9)$ & 10/12(83.3) & $2 / 12(16.7)$ & $11 / 368(3.0)$ \\
\hline Chronic lung disease & $8 / 417(1.9)$ & $7 / 8(87.5)$ & $1 / 8(12.5)$ & $7 / 368(1.9)$ \\
\hline
\end{tabular}

Continuous variables were analyzed using one-way ANOVA test and thecategorical variables were compared using Chisquare test. The deadline of the data collection was by the end of March $7^{\text {th }}$, imported cases from abroad were not 
included in the statistics. ${ }^{* * *} \mathrm{P}<0.001$ vs no-severe group;

\#\#\# $\mathrm{P}<0.001$ vs male; ${ }^{\wedge} \wedge \mathrm{P}<0.01$ vs no Wuhan exposure;

$\$ \$ \$ \mathrm{P}<0.001$ vs no basic diseases.

Table 2. Analysis of 368 discharged patients who infected with COVID-19 in Shenzhen

\begin{tabular}{|c|c|c|c|c|}
\hline $\begin{array}{l}\text { Epidemiological } \\
\text { Characteristics }\end{array}$ & $\begin{array}{l}\text { Dischard / Total } \\
- \text { N., \% }\end{array}$ & $\begin{array}{l}\text { Onset } \\
\text { admission }\end{array}$ & $\begin{array}{l}\text { Time -d } \\
\text { to Admission } \\
\text { to } \\
\text { discharge }\end{array}$ & $\begin{array}{l}\text { Onset } \\
\text { to discharge }\end{array}$ \\
\hline Total & $368 / 417(88.2)$ & $3.8 \pm 3.7$ & $20.8 \pm 7.3$ & $24.6 \pm 8.2$ \\
\hline$\leq 45$ yrs & $180 / 195(92.3)^{*}$ & $3.4 \pm 3.7$ & $18.9 \pm 6.2^{* * *}$ & $22.4 \pm 7.3^{* * *}$ \\
\hline$\geq 46$ yrs & $188 / 222(84.7)$ & $4.2 \pm 3.7$ & $22.6 \pm 7.8$ & $26.8 \pm 8.4$ \\
\hline P value & 0.022 & 0.076 & 0.000 & 0.000 \\
\hline Female & $194 / 219(88.6)$ & $3.6 \pm 3.7$ & $20.6 \pm 7.1$ & $24.2 \pm 8.2$ \\
\hline Male & 174/198(87.9) & $4.1 \pm 3.8$ & $20.9 \pm 7.5$ & $25.1 \pm 8.1$ \\
\hline $\mathrm{P}$ value & 0.879 & 0.169 & 0.691 & 0.324 \\
\hline Wuhan exposure & $196 / 222(88.3)$ & $3.9 \pm 3.8$ & $21.2 \pm 7.7$ & $25.1 \pm 8.1$ \\
\hline No Wuhan exposure & $172 / 195(88.2)$ & $3.7 \pm 3.7$ & $20.2 \pm 6.9$ & $23.9 \pm 8.4$ \\
\hline$P$ value & 1.000 & 0.718 & 0.253 & 0.236 \\
\hline Carried at least one & $108 / 129(83.7)^{\#}$ & $4.2 \pm 3.9$ & $22.2 \pm 7.1^{\#}$ & $26.4 \pm 7.9^{\# \#}$ \\
\hline \multicolumn{5}{|l|}{ basic diseases } \\
\hline No basic diseases & $260 / 288(90.3)$ & $3.7 \pm 3.7$ & $20.2 \pm 7.3$ & $23.9 \pm 8.2$ \\
\hline $\mathrm{P}$ value & 0.014 & 0.183 & 0.018 & 0.006 \\
\hline Severe & $68 / 92(73.9)^{\wedge \wedge}$ & $4.9 \pm 3.8^{\wedge}$ & $26.5 \pm 8.5^{\wedge \wedge}$ & $31.4 \pm 8.5^{\wedge \wedge}$ \\
\hline Non- Severe & 300/325(92.3) & $3.6 \pm 3.6$ & $19.5 \pm 6.3$ & $23.1 \pm 7.2$ \\
\hline $\mathrm{P}$ value & 0.000 & 0.010 & 0.000 & 0.000 \\
\hline
\end{tabular}

Continuous variables were analyzed using one-way ANOVA test and the categorical variables were compared using Chi-square test. The deadline of the data collection was by the end of March $7^{\text {th }}$. ${ }^{*} \mathrm{P}<0.05,{ }^{* * *} \mathrm{P}<0.001$ vs patients $\geq 46$ yrs; $\# \mathrm{P}<0.05$, \#\# $\mathrm{P}<0.01$ vs no basic diseases; $\wedge \mathrm{P}<0.05$, ^^^^ $\mathrm{P}<0.001$ vs no-severe group. 


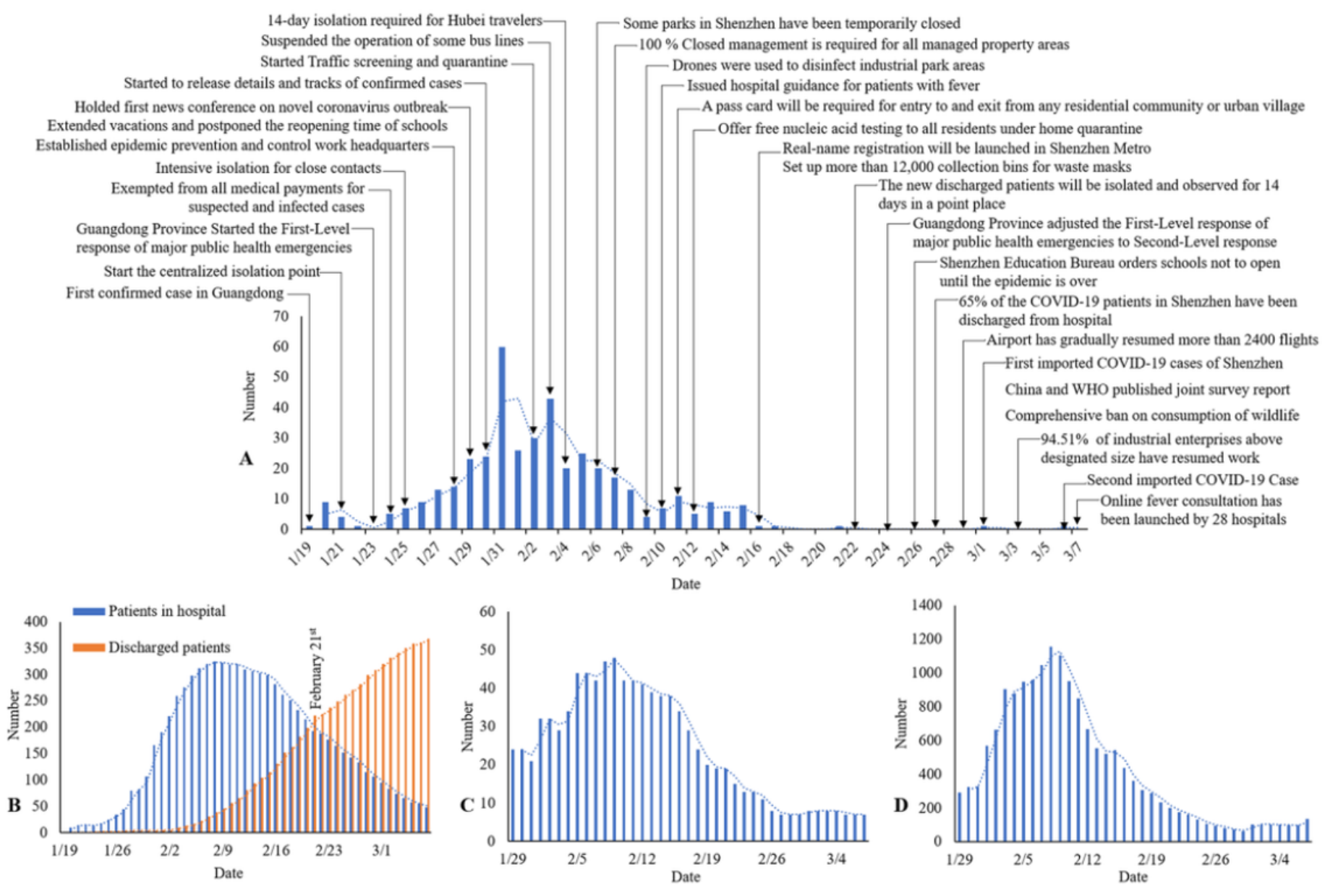

Figure 1

Review of epidemic prevention and control in Shenzhen. (A) Daily progress of the epidemic in Shenzhen and the prevention and control measures. (B) Hospitalized and discharged patients. (C) People in close contact. (D) Hospitalized patients in critical and severe conditions. 

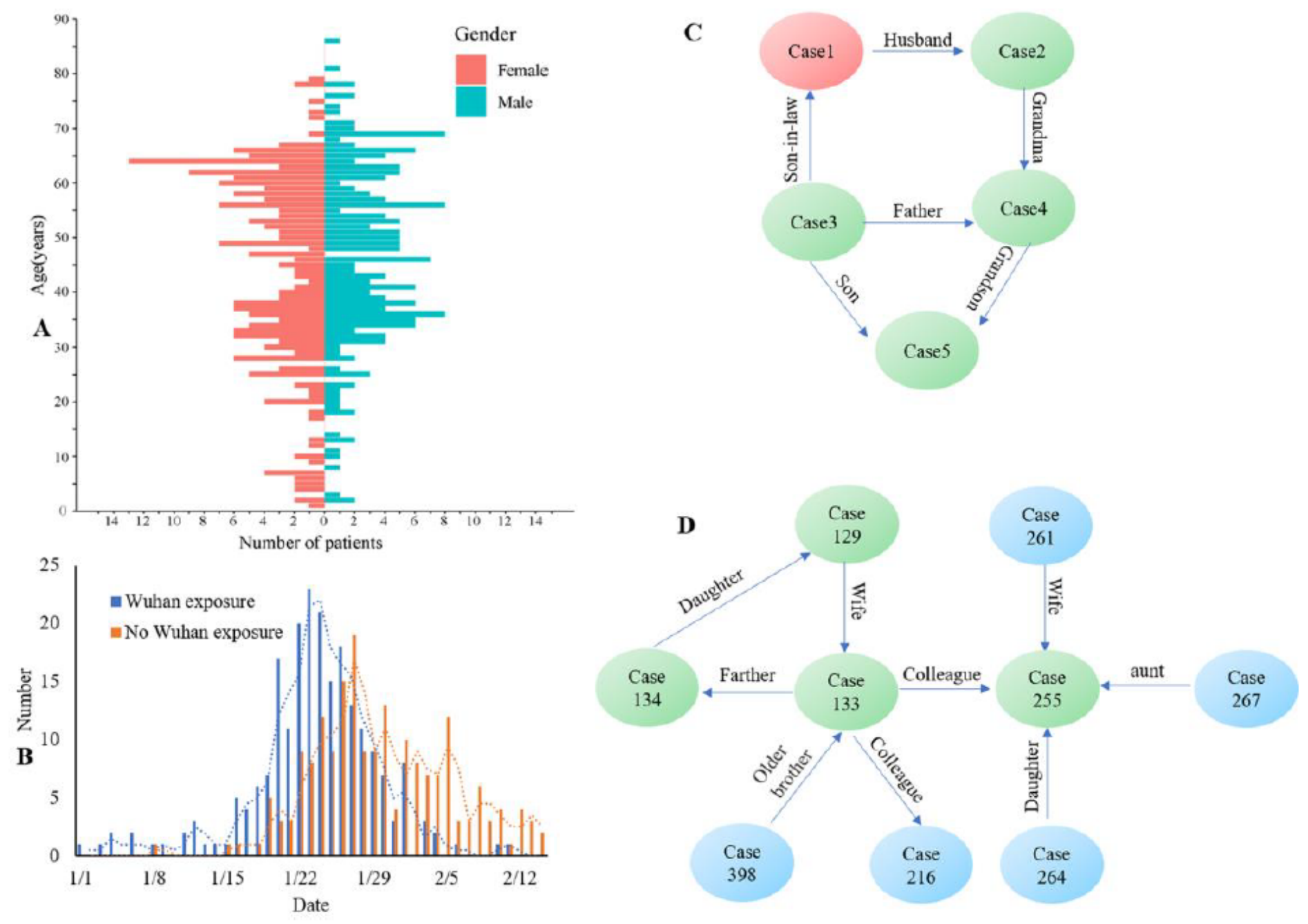

Figure 2

Epidemiologic character of 417 local confirmed COVID-19 cases in Shenzhen. (A) Age and sex patterns. (B) Effects of Wuhan exposure history on onset time. (C) The first family cluster in Shenzhen, case 5 was the first medical patients in Guangdong province and become the key person to find out the first familial cluster; case1 was the first confirmed patients in Guangdong, and died on February 16. In addition to case 5 , the other four have a history of exposure to Wuhan. (D) The biggest cluster in Shenzhen, all the 9 cases have no Wuhan exposure history. Red represents death, green represents discharge, and blue represents hospitalization in (C) and (D). 